Journal of Universal Mathematics

Vol.3 No.1 PP.83-93 (2020)

ISSN-2618-5660

\title{
SOME FIXED POINT THEOREMS FOR CONTRACTIVE MAPPINGS ON ORDERED ORTHOGONAL CONE METRIC SPACES
}

\author{
NURCAN BILGILI GUNGOR AND MEHMET SURMELIOGLU
}

\begin{abstract}
Very recently, orthogonal cone metric spaces, orthogonal completeness and orthogonal continuity are described in [4]. Also some fixed point theorems and their conclusions are proved on orthogonal cone metric spaces in there. In this paper, some fixed point theorems for contractive mappings are presented on ordered orthogonal cone metric spaces. Also an illustrative example is given.
\end{abstract}

\section{Introduction AND PRELIMINARIES}

In 2007, Huang and Zhang [7] introduced cone metric spaces and proved some fixed point theorems of contractive mappings on cone metric spaces. Then, in 2008, Rezapour and Hamlbarani [13] obtained generalizations of some results in [7] by omitting the assumption of normality. Then many researchers are obtained fixed point theorems on cone metric spaces.(see [1],[2],[8],[9],[14],,[15])

On the other hand, in 2017, Gordji et al [5] described the notion of orthogonal set and orthogonal metric spaces. Generalizations of theorems in this field have been considered in some research articles.(see [6],[10],[3],[11])

Very recently, a new concept of orthogonal cone metric spaces, orthogonal completeness and orthogonal continuity are defined in [4]. And illustrative examples are given for this new definitions. Also, an example is given for orthogonal complete cone metric space which is not complete cone metric space. Furthermore, fixed point theorems and their corollaries are proved on orthogonal cone metric spaces.

In this paper, some fixed point theorems for contractive mappings on ordered orthogonal cone metric spaces are presented inspired by [12] and [4]. Also an illustrative example is given.

In the sequel, respectively, $\mathbb{Q}, \mathbb{Q}^{c}, \mathbb{Z}, \mathbb{R}$ denote rational numbers, irrational numbers, integers and real numbers.

Definition 1.1. ([5]) Let $X \neq \emptyset$ and $\perp \subseteq X \times X$ be a binary relation. If $\perp$ satisfies the following condition

$$
\exists x_{0} \in X ;\left(\forall y \in X, y \perp x_{0}\right) \vee\left(\forall y \in X, x_{0} \perp y\right),
$$

2000 Mathematics Subject Classification. 47H10; 54H25.

Key words and phrases. contractive mapping, fixed point, cone metric space, orthogonal cone metric space, ordered metric space. 
it is called an orthogonal set (shortly O-set). And $(X, \perp)$ is called $O$-set. And the element $x_{0}$ is called an orthogonal element.

Example 1.2. ([6]) Let $X=\mathbb{Z}$. Define $m \perp n$ if there exists $k \in \mathbb{Z}$ such that $m=k n$. It is easy to see that $0 \perp n$ for all $n \in \mathbb{Z}$. Hence $(X, \perp)$ is an $O$-set.

By the following example, we can see that $x_{0}$ is not necessarily unique.

Example 1.3. ([6]) Let $X=[0, \infty)$, we define $x \perp y$ if $x y \in\{x, y\}$, then by setting $x_{0}=0$ or $x_{0}=1,(X, \perp)$ is an $O$-set.

Definition 1.4. ([5]) Let $(X, \perp)$ be an orthogonal set $(O$-set). Any two elements $x, y \in X$ are said to be orthogonally related if $x \perp y$.

Definition 1.5. ([5]) A sequence $\left\{x_{n}\right\}$ is called orthogonal sequence (shortly $O$ sequence) if

$$
\left(\forall n \in \mathbb{N} ; x_{n} \perp x_{n+1}\right) \vee\left(\forall n \in \mathbb{N} ; x_{n+1} \perp x_{n}\right) .
$$

Similarly, a Cauchy sequence $\left\{x_{n}\right\}$ is said to be an orthogonally Cauchy sequence (shortly $O$-Cauchy sequence) if

$$
\left(\forall n \in \mathbb{N} ; x_{n} \perp x_{n+1}\right) \vee\left(\forall n \in \mathbb{N} ; x_{n+1} \perp x_{n}\right) .
$$

Definition 1.6. ([5]) Let $(X, \perp)$ be an orthogonal set and $d$ be an usual metric on $X$. Then $(X, \perp, d)$ is called an orthogonal metric space (shortly $O$-metric space).

Definition 1.7. ([7]) Let $E$ be a real Banach space and $P$ a subset of $E . P$ is called a cone if and only if

(i) $P$ is closed,nonempty, $P \neq\left\{\theta_{E}\right\}$,

(ii) $a, b \in \mathbb{R}, a, b \geq 0, x, y \in P \Rightarrow a x+b y \in P$,

(iii) $x \in P$ and $-x \in P \Rightarrow x=\theta_{E}$.

Given a cone $P \subseteq E$, we define a partial ordering $\preceq$ with respect to $P$ by $x \preceq y$ if and only if $y-x \in P$. We shall write $x \prec y$ to indicate that $x \preceq y$ but $x \neq y$, while $x \prec \prec y$ will stand for $y-x \in$ int $P$, int $P$ denotes the interior of $P$.

The cone $P$ is called normal if there is a number $K>0$ such that for all $x, y \in E$, $0 \preceq x \preceq y$ implies $\|x\|_{E} \leq K\|y\|_{E}$.

The least positive number satisfying above is called the normal constant of $P$.

The cone $P$ is called regular if every increasing sequence which is bounded from above is convergent. That is, if $\left\{x_{n}\right\}$ is sequence such that

$$
x_{1} \preceq x_{2} \preceq x_{3} \preceq \ldots \preceq x_{n} \preceq \ldots \preceq y
$$

for some $y \in E$, then there exists $x \in E$ such that $\left\|x_{n}-x\right\|_{E} \rightarrow 0(n \rightarrow \infty)$.

Equivalently the cone $P$ is regular if and only if every decreasing sequence which is bounded from below is convergent.

It is well known that a regular cone is a normal cone.

In the following we always suppose $E$ is a Banach space, $P$ is a cone in $E$ with int $P \neq \emptyset$ and $\preceq$ is partial ordering with respect to $P$. 
Definition 1.8. ([7]) Let $X$ be a nonempty set. Suppose the mapping $d: X \times X \rightarrow$ $E$ satisfies

$\left(d_{1}\right) \theta_{E} \preceq d(x, y)$ for all $x, y \in X$ and $d(x, y)=\theta_{E}$ if and only if $x=y$.

$\left(d_{2}\right) d(x, y)=d(y, x)$ for all $x, y \in X$,

$\left.\left(d_{3}\right) d(x, y) \preceq d / x, z\right)+d(z, y)$ for all $x, y, z \in X$.

Then $d$ is called a cone metric on $X$ and $(X, d)$ is called a cone metric space.

Lemma 1.9. ([14]) Let $(X, d)$ be a cone metric space. Then for each $\theta \prec \prec c$, $c \in E$, there exists $\delta>0$ such that $c-x \in$ intP whenever $\|x\|<\delta, x \in E$.

Definition 1.10. ([4]) Let $(X, \perp)$ be an orthogonal set and $d$ be a cone metric on $X$. Then $(X, \perp, d)$ is called orthogonal cone metric space (briefly $O$-cone metric space).

Example 1.11. ([4]) Let $E=\mathbb{R}^{2}, P=\{(x, y) \in E: x, y \geq 0\} \subseteq \mathbb{R}^{2}$ and $X=\mathbb{Z}$. And $d: X \times X \rightarrow E, d(x, y)=(|x-y|, \alpha|x-y|)$ is defined where $\alpha \geq 0, \alpha \in$ $\mathbb{R}$. Assume that binary relation $\perp$ on $X=\mathbb{Z}$ as Example 1.2 , then $(X, d, \perp)$ is orthogonal cone metric space.

Example 1.12. ([4]) Let $q, b \in \mathbb{R}$ where $q \geq 1, b>1, E=\left\{\left\{x_{n}\right\} \mid x_{n} \in\right.$ $\mathbb{R}$ and $\left.\sum_{n=1}^{\infty}\left(\left|x_{n}\right|\right)^{q}<\infty\right\}$ and $P=\left\{\left\{x_{n}\right\} \in E \mid x_{n} \geq 0, \forall n \in \mathbb{N}\right\}$. Assume that $(X, \perp, \rho)$ is an orthogonal metric space, then the mapping

$$
d: X \times X \rightarrow E, d(x, y)=\left(\frac{\rho}{b^{n}}\right)^{\frac{1}{q}}
$$

can be defined on $X$ and this mapping is an orthogonal cone metric. So $(X, \perp, d)$ is an orthogonal cone metric space.

Example 1.13. ([4]) Let $E=\left(C_{\mathbb{R}}[0, \infty),\|\cdot\|_{\infty}\right)$ and $P=\{f \in \mathbb{E} \mid f(t) \geq 0\}$. Assume that $(X, \perp, \rho)$ is an orthogonal metric space, then the mapping

$$
d: X \times X \rightarrow E, d(x, y)=f_{x, y} \text { where } f_{x, y}(t)=\rho(x, y) t
$$

can be defined on $X$ and this mapping is an orthogonal cone metric. So $(X, \perp, d)$ is an orthogonal cone metric space.

Definition 1.14. ([4]) Let $(X, \perp, d)$ be an $O$-cone metric space. Let $\left\{x_{n}\right\}$ be an $O$-sequence in $X$ and $x \in X$. If for any $c \in E$ with $\theta \prec \prec c$ there is $N \in \mathbb{N}$ such that for all $n \geq N(n \in \mathbb{N}), d\left(x_{n}, x\right) \prec \prec c$, then $O$-sequence $x_{n}$ is said to be convergent and $\left\{x_{n}\right\}$ converges to $x$ ( or $x$ is the limit of $\left\{x_{n}\right\}$ ). We denote this by

$$
\lim _{n \rightarrow \infty} x_{n}=x \text { or } x_{n} \rightarrow x(n \rightarrow \infty) .
$$

Definition 1.15. ([4]) Let $(X, \perp, d)$ be an $O$-cone metric space. Let $\left\{x_{n}\right\}$ be an $O$-sequence in $X$. If for any $c \in E$ with $\theta \prec \prec c$ there is $N \in \mathbb{N}$ such that for all $n, m \geq N(n, m \in \mathbb{N}), d\left(x_{n}, x_{m}\right) \prec \prec c$, then $O$-sequence $x_{n}$ is called an $O$-Cauchy sequence in $X$.

Definition 1.16. ([4]) Let $(X, \perp, d)$ be an $O$-cone metric space, if every $O$-Cauchy sequence in $X$ is convergent in $X$, then $(X, \perp, d)$ is called an $O$-complete cone metric space.

Lemma 1.17. ([4]) Let $(X, \perp, d)$ be an $O$-cone metric space, $\left\{x_{n}\right\}$ be an $O$-sequence in $X .\left\{x_{n}\right\}$ converges to $x \in X$, then $\left\{x_{n}\right\}$ is O-Cauchy sequence. 
Definition 1.18. ([4]) Let $(X, \perp, d)$ be an $O$-cone metric space. If for any $O$ sequence $\left\{x_{n}\right\}$ in $X$, there is an $O$-subsequence $\left\{x_{n_{i}}\right\}$ of $\left\{x_{n}\right\}$ such that $\left\{x_{n_{i}}\right\}$ is convergent in $X$. Then $(X, \perp, d)$ is called a sequently compact $O$-cone metric space.

Definition 1.19. ([4]) Let $(X, \perp, d)$ be an $O$-cone metric space and $\lambda \in \mathbb{R}, 0<$ $\lambda<1$. A mapping $f: X \rightarrow X$ is said to be orthogonal contraction ( shortly $\perp$-contraction ) with Lipschitz constant $\lambda$ when

$$
d(f x, f y) \preceq \lambda d(x, y) \text { if } x \perp y .
$$

Definition 1.20. ([4]) Let $(X, \perp, d)$ be an $O$-cone metric space. A mapping $f$ : $X \rightarrow X$ is called orthogonal preserving ( shortly $\perp$-preserving ) when

$$
f x \perp f y \text { if } x \perp y .
$$

Definition 1.21. ([4]) Let $(X, \perp, d)$ be an $O$-cone metric space. A mapping $f$ : $X \rightarrow X$ is called orthogonal continuous ( shortly $\perp$-continuous ) at $x \in X$ if for each $O$-sequence $\left\{x_{n}\right\}$ in $X$ such that $x_{n} \rightarrow x$ then $f\left(x_{n}\right) \rightarrow f(x)$. Also $f$ is $\perp$-continuous on $X$ if $f$ is $\perp$-continuous in each $x \in X$.

Theorem 1.22. ([4]) Let $(X, \perp, d)$ is an $O$-complete cone metric space (it is not necessarily complete cone metric space $)$ and $\lambda \in \mathbb{R}, 0<\lambda<1$. Let $f:(X, \perp$ $, d) \rightarrow(X, \perp, d)$ is $\perp$-contraction with Lipschitz constant $\lambda$ and $\perp$-preserving. In this case, there exists a point $x^{*} \in X$ such that for any orthogonal element $x_{0} \in X$, the iteration sequence $\left\{f^{n}\left(x_{0}\right)\right\}$ converges to this point. Also, if $f$ is $\perp$-continuous at $x^{*} \in X$, then $x^{*} \in X$ is a unique fixed point of $f$.

Corollary 1.23. ([4]) Let $(X, \perp, d)$ is an $O$-complete cone metric space, $P$ be a normal cone with normal constant $K$ and $\lambda \in \mathbb{R}, 0<\lambda<1$. For $c \in E$ with $0 \prec \prec c$ and any $x_{0} \in X$, define $B\left(x_{0}, c\right)=\left\{x \in X: d\left(x_{0}, x\right) \leq c\right\}$. Let $f:(X, \perp$ $, d) \rightarrow(X, \perp, d)$ is $\perp$-contraction with Lipschitz constant $\lambda$ for all $x, y \in B\left(x_{0}, c\right)$, $\perp$-preserving on $B\left(x_{0}, c\right)$ and $d\left(f x_{0}, x_{0}\right) \preceq(1-k) c$. In this case, there exists a point $x^{*} \in B\left(x_{0}, c\right)$ such that for any orthogonal element $x_{0} \in X$, the iteration sequence $\left\{f^{n}\left(x_{0}\right)\right\}$ converges to this point. Also, if $f$ is $\perp$-continuous on $B\left(x_{0}, c\right)$, then $x^{*} \in B\left(x_{0}, c\right)$ is a unique fixed point of $f$.

\section{Main Results}

Now, we are ready to give and prove our main results.

Theorem 2.1. Let $(X, \sqsubseteq)$ be a partially ordered set, $(X, \perp, d)$ is an $O$-complete cone metric space, $f:(X, \perp, d) \rightarrow(X, \perp, d)$ is nondecreasing mapping with respect to $\sqsubseteq$ and $\perp$-preserving. And suppose that there exists $\lambda \in \mathbb{R}, 0<\lambda<1$ such that $d(f x, f y) \preceq \lambda d(x, y)$ for each orthogonally related $x, y \in X$ with $y \sqsubseteq x$. Assume that there exists an orthogonal element $x_{0} \in X$ such that $x_{0} \sqsubseteq f x_{0}$ then the iteration sequence $\left\{f^{n}\left(x_{0}\right)\right\}$ converges to a point $x^{*} \in X$. Also, if $f$ is $\perp$-continuous at $x^{*} \in X$, then $x^{*} \in X$ is a fixed point of $f$.

Proof. Because of $(X, \perp)$ is an $O$-set there exists an orthogonal element in $x_{0} \in X$. From the assumption choosing such that $x_{0} \sqsubseteq f x_{0}$ does not disrupt generality. Thus from $\mathrm{f}$ is a self mapping on $X$, for this orthogonal element $x_{0} \in X, x_{1} \in X$ can be chosen as $x_{1}=f\left(x_{0}\right)$. Thus,

$$
\begin{aligned}
& x_{0} \perp f\left(x_{0}\right) \vee f\left(x_{0}\right) \perp x_{0} \\
\Rightarrow & x_{0} \perp x_{1} \vee x_{1} \perp x_{0} .
\end{aligned}
$$


Then, if we continue in the same way

$$
x_{1}=f\left(x_{0}\right), x_{2}=f\left(x_{1}\right)=f^{2}\left(x_{0}\right), \ldots, x_{n}=f\left(x_{n-1}\right)=f^{n}\left(x_{0}\right)
$$

so $\left\{f^{n}\left(x_{0}\right)\right\}$ is an iteration sequence. Since $f$ is $\perp$-preserving, $\left\{f^{n}\left(x_{0}\right)\right\}$ is an $O$ sequence. Using $x_{0} \sqsubseteq f x_{0}$ and $f$ is nondecreasing mapping with respect to $\sqsubseteq$, we get

$$
x_{0} \sqsubseteq f\left(x_{0}\right) \sqsubseteq f^{2}\left(x_{0}\right) \sqsubseteq \cdots \sqsubseteq f^{n}\left(x_{0}\right) \sqsubseteq \cdots
$$

In this case, for all $n \in \mathbb{N}$,

$$
\begin{aligned}
d\left(x_{n+1}, x_{n}\right) & =d\left(f\left(x_{n}\right), f\left(x_{n-1}\right)\right) \\
& \preceq \lambda d\left(x_{n}, x_{n-1}\right) \\
& \preceq \ldots \\
& \preceq \lambda^{n} d\left(x_{1}, x_{0}\right) .
\end{aligned}
$$

If any $n \in \mathbb{N}, x_{n}=x_{n+1}$ then we get $x_{n}=f\left(x_{n}\right)$ and so $f$ has a fixed point. Assume that $\forall n, n+1 \in \mathbb{N}, x_{n} \neq x_{n+1}$. In this case, $\forall n, m \in \mathbb{N}, n>m$,

$$
\begin{aligned}
\theta \preceq d\left(x_{n}, x_{m}\right) & \preceq d\left(x_{n}, x_{n-1}\right)+d\left(x_{n-1}, x_{n-2}\right)+\ldots+d\left(x_{m+1}, x_{m}\right) \\
& \preceq \lambda^{n-1} d\left(x_{1}, x_{0}\right)+\lambda^{n-2} d\left(x_{1}, x_{0}\right)+\ldots+\lambda^{m} d\left(x_{1}, x_{0}\right) \\
& \preceq \frac{\lambda^{m}}{1-\lambda} d\left(x_{1}, x_{0}\right) .
\end{aligned}
$$

In the sequel there are two cases:

Case I: If $P$ is normal cone with normal constant $K$, from the inequality 2.33 ,

$$
\begin{aligned}
\left\|d\left(x_{n}, x_{m}\right)\right\| & \leq K\left\|\frac{\lambda^{m}}{1-\lambda} d\left(x_{1}, x_{0}\right)\right\| \\
& \leq \frac{\lambda^{m}}{1-\lambda} K\left\|d\left(x_{1}, x_{0}\right)\right\|
\end{aligned}
$$

Using the above equation, since $0<\lambda<1, d\left(x_{n}, x_{m}\right) \rightarrow \theta(n, m \rightarrow \infty)$ and so $\left\{x_{n}\right\}=\left\{f^{n}\left(x_{0}\right)\right\}$ is an $O$-Cauchy sequence.

Case II: If $P$ is not normal cone, let $c \in E$ such that $\theta \prec \prec c$. Then $c \in$ int $P$. Also $\delta>0$ can be chosen such that $c+N_{\delta}(\theta) \subset P$ where $N_{\delta}(\theta)=\{y \in E:\|y-\theta\|<\delta\}$. Since $0<\lambda<1$,

$$
\left\|\frac{\lambda^{m}}{1-\lambda} d\left(x_{1}, x_{0}\right)\right\|=\frac{\lambda^{m}}{1-\lambda}\left\|d\left(x_{1}, x_{0}\right)\right\| \rightarrow 0(m \rightarrow \infty) .
$$

From the choosing of $\delta,\left\|\frac{\lambda^{m}}{1-\lambda} d\left(x_{1}, x_{0}\right)\right\|<\delta$ and using the Lemma 1.9 we get

$$
c-\frac{\lambda^{m}}{1-\lambda} d\left(x_{1}, x_{0}\right) \in \operatorname{int} P \text { that is } \frac{\lambda^{m}}{1-\lambda} d\left(x_{1}, x_{0}\right) \prec \prec c(m \rightarrow \infty) .
$$

Thus, for all $n, m \in \mathbb{N}$ such that $n \geq m$, we obtain that $d\left(x_{n}, x_{m}\right) \leq \frac{\lambda^{m}}{1-\lambda} d\left(x_{1}, x_{0}\right) \prec \prec$ $c$ so $\left\{x_{n}\right\}=\left\{f^{n}\left(x_{0}\right)\right\}$ is an $O$-Cauchy sequence.

In both cases, since $(X, \perp, d)$ is an $O$-complete cone metric space, there exists $x^{*} \in X$ such that $\left\{x_{n}\right\}=\left\{f^{n}\left(x_{0}\right)\right\}$ converges to this point. Now, assume that $f$ is $\perp$-continuous at $x^{*} \in X$ and let $c \in E$ such that $\theta \prec \prec c$. Because of $\left\{x_{n}\right\}=$ $\left\{f^{n}\left(x_{0}\right)\right\}$ converges to $x^{*} \in X$ and $f$ is $\perp$-continuous at $x^{*} \in X$, there exists $n_{0} \in \mathbb{N}$ and for all $n \in \mathbb{N}$ such that $n \geq n_{0}$,

$$
d\left(x_{n+1}, x^{*}\right) \prec \prec \frac{c}{2} \text { and } d\left(f x_{n}, f x^{*}\right) \prec \prec \frac{c}{2} .
$$

And so for all $n \in \mathbb{N}$ such that $n \geq n_{0}, d\left(f x^{*}, x^{*}\right) \preceq d\left(f x^{*}, f x_{n}\right)+d\left(f x_{n}, x^{*}\right) \prec \prec c$. On the other hand, for $m \in \mathbb{N}, m \geq 1$ we obtain $0<\frac{1}{m} \leq 1$. Using $c \in$ int $P$ and $\lambda i n t P \subseteq \operatorname{int} P(\lambda \in \mathbb{R}, \lambda>0)$ we get $\frac{c}{m} \in \operatorname{int} P$. Thus, for all $n \in \mathbb{N}$ such that $n \geq n_{0}$ and for $m \in \mathbb{N}, m \geq 1$ we hold $d\left(f x^{*}, x^{*}\right) \prec \prec \frac{c}{m}$, then $\frac{c}{m}-d\left(f x^{*}, x^{*}\right) \in P$. Using the 
cone $P$ is closed set, where taking limit $m \rightarrow \infty$ we get $\lim _{m \rightarrow \infty}\left(\frac{c}{m}-d\left(f x^{*}, x^{*}\right)=\right.$ $-d\left(f x^{*}, x^{*}\right) \in P$. Besides $\theta \preceq d\left(f x^{*}, x^{*}\right)$ that is $d\left(f x^{*}, x^{*}\right) \in P$. So, because of $P$ is cone $d\left(f x^{*}, x^{*}\right)=\theta$ that is $f x^{*}=x^{*}$, so $x^{*} \in X$ is a fixed point of $f$.

Remark 2.2. In the above theorem, because of $(X, \perp)$ is an $O$-set,

$$
\exists x_{0} \in X ;\left(\forall y \in X, y \perp x_{0}\right) \vee\left(\forall y \in X, x_{0} \perp y\right) .
$$

But, $x_{0} \sqsubseteq f x_{0}$ may not be provided for any orthogonal element of $X$ and in this case, we can alternatively give the following theorem.

Theorem 2.3. Let $(X, \sqsubseteq)$ be a partially ordered set, $(X, \perp, d)$ is an O-complete cone metric space, $f:(X, \perp, d) \rightarrow(X, \perp, d)$ is nondecreasing mapping with respect to $\sqsubseteq$ and $\perp$-preserving. Also suppose that there exists $\lambda \in \mathbb{R}, 0<\lambda<1$ such that $d(f x, f y) \preceq \lambda d(x, y)$ for each orthogonally related $x, y \in X$ with $y \sqsubseteq x$. Assume that there exists a $y_{0} \in X$ such that $y_{0} \sqsubseteq f y_{0}$ and $y_{0}, f y_{0}$ are orthogonally related elements. In this case, the iteration sequence $\left\{f^{n}\left(y_{0}\right)\right\}$ converges to a point $x^{*} \in X$. Also, if $f$ is $\perp$-continuous at $x^{*} \in X$, then $x^{*} \in X$ is a fixed point of $f$.

Proof. From the assumption there exists a $y_{0} \in X$ such that $y_{0} \sqsubseteq f y_{0}$ and $y_{0}, f y_{0}$ are orthogonally related elements. Thus from $\mathrm{f}$ is a self mapping on $X$, for this element $y_{0} \in X, y_{1} \in X$ can be chosen as $y_{1}=f\left(y_{0}\right)$. Thus,

$$
\begin{aligned}
& y_{0} \perp f\left(y_{0}\right) \vee f\left(y_{0}\right) \perp y_{0} \\
\Rightarrow y_{0} & \perp y_{1} \vee y_{1} \perp y_{0} .
\end{aligned}
$$

Then, if we continue in the same way

$$
y_{1}=f\left(y_{0}\right), y_{2}=f\left(y_{1}\right)=f^{2}\left(y_{0}\right), \ldots, y_{n}=f\left(y_{n-1}\right)=f^{n}\left(y_{0}\right)
$$

so $\left\{f^{n}\left(y_{0}\right)\right\}$ is an iteration sequence. Since $f$ is $\perp$-preserving, $\left\{f^{n}\left(y_{0}\right)\right\}$ is an $O$ sequence. Using $y_{0} \sqsubseteq f y_{0}$ and $f$ is nondecreasing mapping with respect to $\sqsubseteq$, we get

$$
y_{0} \sqsubseteq f\left(y_{0}\right) \sqsubseteq f^{2}\left(y_{0}\right) \sqsubseteq \cdots \sqsubseteq f^{n}\left(y_{0}\right) \sqsubseteq \cdots
$$

In this case, for all $n \in \mathbb{N}$,

$$
\begin{aligned}
d\left(y_{n+1}, y_{n}\right) & =d\left(f\left(y_{n}\right), f\left(y_{n-1}\right)\right) \\
& \preceq \lambda d\left(y_{n}, y_{n-1}\right) \\
& \preceq \ldots \\
& \preceq \lambda^{n} d\left(y_{1}, y_{0}\right) .
\end{aligned}
$$

If any $n \in \mathbb{N}, y_{n}=y_{n+1}$ then we get $y_{n}=f\left(y_{n}\right)$ and so $f$ has a fixed point. Assume that $\forall n, n+1 \in \mathbb{N}, y_{n} \neq y_{n+1}$. In this case, $\forall n, m \in \mathbb{N}, n>m$,

$$
\begin{aligned}
\theta \preceq d\left(y_{n}, y_{m}\right) & \preceq d\left(y_{n}, y_{n-1}\right)+d\left(y_{n-1}, y_{n-2}\right)+\ldots+d\left(y_{m+1}, y_{m}\right) \\
& \preceq \lambda^{n-1} d\left(y_{1}, y_{0}\right)+\lambda^{n-2} d\left(y_{1}, y_{0}\right)+\ldots+\lambda^{m} d\left(y_{1}, y_{0}\right) \\
& \preceq \frac{\lambda^{m}}{1-\lambda} d\left(y_{1}, y_{0}\right) .
\end{aligned}
$$

In the sequel there are two cases:

Case I: If $P$ is normal cone with normal constant $K$, from the inequality 2.15 ,

$$
\begin{aligned}
\left\|d\left(y_{n}, y_{m}\right)\right\| & \leq K\left\|\frac{\lambda^{m}}{1-\lambda} d\left(y_{1}, y_{0}\right)\right\| \\
& \leq \frac{\lambda^{m}}{1-\lambda} K\left\|d\left(y_{1}, y_{0}\right)\right\|
\end{aligned}
$$

Using the above equation, since $0<\lambda<1, d\left(y_{n}, y_{m}\right) \rightarrow \theta(n, m \rightarrow \infty)$ and so $\left\{y_{n}\right\}=\left\{f^{n}\left(y_{0}\right)\right\}$ is an $O$-Cauchy sequence. 
Case II: If $P$ is not normal cone, let $c \in E$ such that $\theta \prec \prec c$. Then $c \in$ int $P$. Also $\delta>0$ can be chosen such that $c+N_{\delta}(\theta) \subset P$ where $N_{\delta}(\theta)=\{y \in E:\|y-\theta\|<\delta\}$. Since $0<\lambda<1$,

$$
\left\|\frac{\lambda^{m}}{1-\lambda} d\left(y_{1}, y_{0}\right)\right\|=\frac{\lambda^{m}}{1-\lambda}\left\|d\left(y_{1}, y_{0}\right)\right\| \rightarrow 0(m \rightarrow \infty) .
$$

From the choosing of $\delta,\left\|\frac{\lambda^{m}}{1-\lambda} d\left(y_{1}, y_{0}\right)\right\|<\delta$ and using the Lemma 1.9 we get

$$
c-\frac{\lambda^{m}}{1-\lambda} d\left(y_{1}, y_{0}\right) \in \operatorname{intP} \text { that is } \frac{\lambda^{m}}{1-\lambda} d\left(y_{1}, y_{0}\right) \prec \prec c(m \rightarrow \infty) .
$$

Thus, for all $n, m \in \mathbb{N}$ such that $n \geq m$, we obtain that $d\left(y_{n}, y_{m}\right) \leq \frac{\lambda^{m}}{1-\lambda} d\left(y_{1}, y_{0}\right) \prec \prec$ $c$ so $\left\{y_{n}\right\}=\left\{f^{n}\left(y_{0}\right)\right\}$ is an $O$-Cauchy sequence.

In both cases, since $(X, \perp, d)$ is an $O$-complete cone metric space, there exists $x^{*} \in X$ such that $\left\{y_{n}\right\}=\left\{f^{n}\left(y_{0}\right)\right\}$ converges to this point. Now, assume that $f$ is $\perp$-continuous at $x^{*} \in X$ and let $c \in E$ such that $\theta \prec \prec c$. Because of $\left\{y_{n}\right\}=$ $\left\{f^{n}\left(y_{0}\right)\right\}$ converges to $x^{*} \in X$ and $f$ is $\perp$-continuous at $x^{*} \in X$, there exists $n_{0} \in \mathbb{N}$ and for all $n \in \mathbb{N}$ such that $n \geq n_{0}$,

$$
d\left(y_{n+1}, x^{*}\right) \prec \prec \frac{c}{2} \text { and } d\left(f y_{n}, f x^{*}\right) \prec \prec \frac{c}{2} .
$$

And so for all $n \in \mathbb{N}$ such that $n \geq n_{0}, d\left(f x^{*}, x^{*}\right) \preceq d\left(f x^{*}, f y_{n}\right)+d\left(f y_{n}, x^{*}\right) \prec \prec c$. On the other hand, for $m \in \mathbb{N}, m \geq 1$ we obtain $0<\frac{1}{m} \leq 1$. Using $c \in$ int $P$ and $\lambda$ int $P \subseteq \operatorname{int} P(\lambda \in \mathbb{R}, \lambda>0)$ we get $\frac{c}{m} \in \operatorname{int} P$. Thus, for all $n \in \mathbb{N}$ such that $n \geq n_{0}$ and for $m \in \mathbb{N}, m \geq 1$ we hold $d\left(f x^{*}, x^{*}\right) \prec \prec \frac{c}{m}$, then $\frac{c}{m}-d\left(f x^{*}, x^{*}\right) \in P$. Using the cone $P$ is closed set, where taking limit $m \rightarrow \infty$ we get $\lim _{m \rightarrow \infty}\left(\frac{c}{m}-d\left(f x^{*}, x^{*}\right)=\right.$ $-d\left(f x^{*}, x^{*}\right) \in P$. Besides $\theta \preceq d\left(f x^{*}, x^{*}\right)$ that is $d\left(f x^{*}, x^{*}\right) \in P$. So, because of $P$ is cone $d\left(f x^{*}, x^{*}\right)=\theta$ that is $f x^{*}=x^{*}$, so $x^{*} \in X$ is a fixed point of $f$.

Example 2.4. Let $E=\mathbb{R}^{2}$ be the Euclidean plane, $P=\{(x, y) \in E: x, y \geq 0\}$ be a cone in $E, X=\{(x, 0) \in E: 0 \leq x, y \leq 1\}$ and consider the following relation on $X,(X, \sqsubseteq)$ be a partially ordered set.

$$
(x, 0) \sqsubseteq(y, 0) \Longleftrightarrow(x=y) \vee(x \leq y, \forall x, y \in[0,1] .
$$

Also, define the binary relation $\perp$ on $X$ such that

$$
(x, 0) \perp(y, 0) \Longleftrightarrow x y \leq \min \{x, y\} .
$$

In this case, $(X, \perp)$ is an O-set. The mapping $d: X \times X \rightarrow E$ is defined by

$$
d((x, 0),(y, 0))=\left(\frac{4}{5}|x-y|,|x-y|\right) .
$$

Then, $(X, \perp, d)$ is an $O$-complete cone metric space. Let mapping $f:(X, \perp, d) \rightarrow$ $(X, \perp, d)$ with

$$
f((x, 0))=\left(\frac{x+1}{2}, 0\right)
$$

Then, $f$ is $\perp$-preserving, nondecreasing mapping with respect to $\sqsubseteq$ and $\perp$-continuous on $X$. Also, there exists $\lambda_{0}=\frac{3}{4} \in \mathbb{R}, 0<\lambda_{0}<1$ such that $d(f((x, 0)), f((y, 0))) \preceq$ $\lambda_{0} d(((x, 0)),((y, 0)))$ for each orthogonally related $(x, 0),(y, 0) \in X$ with $(y, 0) \sqsubseteq$ $(x, 0)$. And there exists an orthogonal element $\left(\frac{1}{3}, 0\right) \in X$ such that $\left(\frac{1}{3}, 0\right) \sqsubseteq$ $f\left(\left(\frac{1}{3}, 0\right)\right)$. Thus, all hypothesis of Theorem 2.1 satisfy and so, it is obvious that $\left\{f^{n}\left(\left(\frac{1}{3}, 0\right)\right)\right\}$ converges to a point $(1,0) \in X$. Due to the $\perp$-continuity of $f$ on $X$, $(1,0)$ is a fixed point of $f$. 
Now, we can give the following theorem which is obtained by adding a new condition instead of continuity of $f$ in Theorem 2.1.

Theorem 2.5. Let $(X, \sqsubseteq)$ be a partially ordered set, $(X, \perp, d)$ is an O-complete cone metric space, $f:(X, \perp, d) \rightarrow(X, \perp, d)$ is nondecreasing mapping with respect to $\sqsubseteq$ and $\perp$-preserving. Also suppose that the following conditions hold:

(i) there exists $\lambda \in \mathbb{R}, 0<\lambda<1$ such that $d(f x, f y) \preceq \lambda d(x, y)$ for each orthogonally related $x, y \in X$ with $y \sqsubseteq x$.

(ii) there exists an orthogonal element $x_{0} \in X$ such that $x_{0} \sqsubseteq f x_{0}$,

(iii) if an increasing sequence $\left\{x_{n}\right\}$ converges to $x \in X$, then $x_{n} \sqsubseteq x$ and $x_{n}, x^{*}$ are orthogonally related elements for all $n$.

In this case, the iteration sequence $\left\{f^{n}\left(x_{0}\right)\right\}$ converges to a point $x^{*} \in X$ and $x^{*} \in X$ is a fixed point of $f$.

Proof. As the proof of Theorem 2.1, there exists $x^{*} \in X$ such that $\left\{x_{n}\right\}=\left\{f^{n}\left(x_{0}\right)\right\}$ converges to this point. Now, from the condition (iii) $f^{n}\left(x_{0}\right) \sqsubseteq x^{*}$ and $f^{n}\left(x_{0}\right), x^{*}$ are orthogonally related elements for all $n$. Thus, from the condition $(i)$,

$$
\begin{aligned}
d\left(f x^{*}, x^{*}\right) & \preceq d\left(f x^{*}, f^{n+1} x_{0}\right)+d\left(f^{n+1} x_{0}, x^{*}\right) \\
& \preceq \lambda d\left(x^{*}, f^{n} x_{0}\right)+d\left(f^{n+1} x_{0}, x^{*}\right) .
\end{aligned}
$$

Let $c \in E$ such that $\theta \prec \prec c$. Because of $\left\{x_{n}\right\}=\left\{f^{n}\left(x_{0}\right)\right\}$ converges to $x^{*} \in X$, there exists $n_{0} \in \mathbb{N}$ and for all $n \in \mathbb{N}$ such that $n \geq n_{0}$,

$$
d\left(x_{n}, x^{*}\right) \prec \prec \frac{c}{2 \lambda} \text { and } d\left(x_{n+1}, x^{*}\right) \prec \prec \frac{c}{2} .
$$

And so for all $n \in \mathbb{N}$ such that $n \geq n_{0}, d\left(f x^{*}, x^{*}\right) \prec \prec c$. On the other hand, for $m \in$ $\mathbb{N}, m \geq 1$ we obtain $0<\frac{1}{m} \leq 1$. Using $c \in$ int $P$ and $\lambda$ int $P \subseteq$ int $P(\lambda \in \mathbb{R}, \lambda>0)$ we get $\frac{c}{m} \in$ int $P$. Thus, for all $n \in \mathbb{N}$ such that $n \geq n_{0}$ and for $m \in \mathbb{N}, m \geq 1$ we hold $d\left(f x^{*}, x^{*}\right) \prec \prec \frac{c}{m}$, then $\frac{c}{m}-d\left(f x^{*}, x^{*}\right) \in P$. Using the cone $P$ is closed set, where taking limit $m \rightarrow \infty$ we get $\lim _{m \rightarrow \infty}\left(\frac{c}{m}-d\left(f x^{*}, x^{*}\right)=-d\left(f x^{*}, x^{*}\right) \in P\right.$. Besides $\theta \preceq d\left(f x^{*}, x^{*}\right)$ that is $d\left(f x^{*}, x^{*}\right) \in P$. So, because of $P$ is cone $d\left(f x^{*}, x^{*}\right)=\theta$ that is $f x^{*}=x^{*}$.

The generalization of Theorem 2.1 can be given as follows:

Theorem 2.6. Let $(X, \sqsubseteq)$ be a partially ordered set, $(X, \perp, d)$ is an $O$-complete cone metric space, $f:(X, \perp, d) \rightarrow(X, \perp, d)$ is nondecreasing mapping with respect to $\sqsubseteq$ and $\perp$-preserving. Also suppose that the following conditions hold:

(i) there exists $\alpha, \beta, \gamma \in \mathbb{R}, 0 \leq \alpha, \beta, \gamma$ and $\alpha+2 \beta+2 \gamma<1$ such that

$$
d(f x, f y) \preceq \alpha d(x, y)+\beta[d(x, f x)+d(y, f y)]+\gamma[d(x, f y)+d(y, f x)]
$$

for each orthogonally related $x, y \in X$ with $y \sqsubseteq x$,

(ii) there exists an orthogonal element $x_{0} \in X$ such that $x_{0} \sqsubseteq f x_{0}$.

In this case, the iteration sequence $\left\{f^{n}\left(x_{0}\right)\right\}$ converges to a point $x^{*} \in X$ and if $f$ is $\perp$-continuous at $x^{*} \in X$, then $x^{*} \in X$ is a fixed point of $f$.

Proof. Because of $(X, \perp)$ is an $O$-set there exists an orthogonal element in $x_{0} \in X$. From the assumption choosing such that $x_{0} \sqsubseteq f x_{0}$ does not disrupt generality. Thus from $\mathrm{f}$ is a self mapping on $X$, for this orthogonal element $x_{0} \in X, x_{1} \in X$ can be chosen as $x_{1}=f\left(x_{0}\right)$. Thus,

$$
\begin{aligned}
& x_{0} \perp f\left(x_{0}\right) \vee f\left(x_{0}\right) \perp x_{0} \\
\Rightarrow & x_{0} \perp x_{1} \vee x_{1} \perp x_{0} .
\end{aligned}
$$


Then, if we continue in the same way

$$
x_{1}=f\left(x_{0}\right), x_{2}=f\left(x_{1}\right)=f^{2}\left(x_{0}\right), \ldots, x_{n}=f\left(x_{n-1}\right)=f^{n}\left(x_{0}\right)
$$

so $\left\{f^{n}\left(x_{0}\right)\right\}$ is an iteration sequence. Since $f$ is $\perp$-preserving, $\left\{f^{n}\left(x_{0}\right)\right\}$ is an $O$ sequence. Using $x_{0} \sqsubseteq f x_{0}$ and $f$ is nondecreasing mapping with respect to $\sqsubseteq$, we get

$$
x_{0} \sqsubseteq f\left(x_{0}\right) \sqsubseteq f^{2}\left(x_{0}\right) \sqsubseteq \cdots \sqsubseteq f^{n}\left(x_{0}\right) \sqsubseteq \cdots
$$

In this case, for all $n \in \mathbb{N}$,

$$
\begin{aligned}
d\left(x_{n+1}, x_{n}\right) & =d\left(f\left(x_{n}\right), f\left(x_{n-1}\right)\right) \\
& \preceq \alpha d\left(x_{n}, x_{n-1}\right)+\beta\left[d\left(x_{n}, f x_{n}\right)+d\left(x_{n-1}, f x_{n-1}\right)\right]+\gamma\left[d\left(x_{n}, f x_{n-1}\right)+d\left(x_{n-1}, f x_{n}\right)\right] \\
& =\alpha d\left(x_{n}, x_{n-1}\right)+\beta\left[d\left(x_{n}, x_{n+1}\right)+d\left(x_{n-1}, x_{n}\right)\right]+\gamma\left[d\left(x_{n}, x_{n}\right)+d\left(x_{n-1}, x_{n+1}\right)\right] \\
& \preceq \alpha d\left(x_{n}, x_{n-1}\right)+\beta\left[d\left(x_{n}, x_{n+1}\right)+d\left(x_{n-1}, x_{n}\right)\right]+\gamma\left[d\left(x_{n-1}, x_{n}\right)+d\left(x_{n}, x_{n+1}\right)\right] .
\end{aligned}
$$

And so, we get

$$
d\left(x_{n+1}, x_{n}\right) \preceq \frac{\alpha+\beta+\gamma}{1-\beta-\gamma} d\left(x_{n}, x_{n-1}\right)
$$

Since $\alpha, \beta, \gamma \in \mathbb{R}, 0 \leq \alpha, \beta, \gamma$ and $\alpha+2 \beta+2 \gamma<1$, if $t=\frac{\alpha+\beta+\gamma}{1-\beta-\gamma}$ is chosen then $t \in(0,1)$ and

$$
\begin{aligned}
d\left(x_{n+1}, x_{n}\right) & \preceq t d\left(x_{n}, x_{n-1}\right) \\
& \preceq t^{2} d\left(x_{n-1}, x_{n-2}\right) \\
& \preceq \ldots \\
& \preceq t^{n} d\left(x_{1}, x_{0}\right) .
\end{aligned}
$$

If any $n \in \mathbb{N}, x_{n}=x_{n+1}$ then we get $x_{n}=f\left(x_{n}\right)$ and so $f$ has a fixed point. Assume that $\forall n, n+1 \in \mathbb{N}, x_{n} \neq x_{n+1}$. In this case, $\forall n, m \in \mathbb{N}, n>m$,

$$
\begin{aligned}
\theta \preceq d\left(x_{n}, x_{m}\right) & \preceq d\left(x_{n}, x_{n-1}\right)+d\left(x_{n-1}, x_{n-2}\right)+\ldots+d\left(x_{m+1}, x_{m}\right) \\
& \preceq t^{n-1} d\left(x_{1}, x_{0}\right)+t^{n-2} d\left(x_{1}, x_{0}\right)+\ldots+t^{m} d\left(x_{1}, x_{0}\right) \\
& \preceq \frac{t^{m}}{1-t} d\left(x_{1}, x_{0}\right) .
\end{aligned}
$$

In the sequel there are two cases:

Case I: If $P$ is normal cone with normal constant $K$, from the inequality 2.33 ,

$$
\begin{aligned}
\left\|d\left(x_{n}, x_{m}\right)\right\| & \leq K\left\|\frac{t^{m}}{1-t} d\left(x_{1}, x_{0}\right)\right\| \\
& \leq \frac{t^{m}}{1-t} K\left\|d\left(x_{1}, x_{0}\right)\right\|
\end{aligned}
$$

Using the above equation, since $0<t<1, d\left(x_{n}, x_{m}\right) \rightarrow \theta(n, m \rightarrow \infty)$ and so $\left\{x_{n}\right\}=\left\{f^{n}\left(x_{0}\right)\right\}$ is an $O$-Cauchy sequence.

Case II: If $P$ is not normal cone, let $c \in E$ such that $\theta \prec \prec c$. Then $c \in$ int $P$. Also $\delta>0$ can be chosen such that $c+N_{\delta}(\theta) \subset P$ where $N_{\delta}(\theta)=\{y \in E:\|y-\theta\|<\delta\}$.

Since $0<t<1$,

$$
\left\|\frac{t^{m}}{1-t} d\left(x_{1}, x_{0}\right)\right\|=\frac{t^{m}}{1-t}\left\|d\left(x_{1}, x_{0}\right)\right\| \rightarrow 0(m \rightarrow \infty) .
$$

From the choosing of $\delta,\left\|\frac{t^{m}}{1-t} d\left(x_{1}, x_{0}\right)\right\|<\delta$ and using the Lemma 1.9 we get

$$
c-\frac{t^{m}}{1-t} d\left(x_{1}, x_{0}\right) \in \operatorname{intP} \text { that is } \frac{t^{m}}{1-t} d\left(x_{1}, x_{0}\right) \prec \prec c(m \rightarrow \infty) \text {. }
$$

Thus, for all $n, m \in \mathbb{N}$ such that $n \geq m$, we obtain that $d\left(x_{n}, x_{m}\right) \leq \frac{t^{m}}{1-t} d\left(x_{1}, x_{0}\right) \prec \prec$ $c$ so $\left\{x_{n}\right\}=\left\{f^{n}\left(x_{0}\right)\right\}$ is an $O$-Cauchy sequence. 
In both cases, since $(X, \perp, d)$ is an $O$-complete cone metric space, there exists $x^{*} \in X$ such that $\left\{x_{n}\right\}=\left\{f^{n}\left(x_{0}\right)\right\}$ converges to this point. Now, assume that $f$ is $\perp$-continuous at $x^{*} \in X$ and let $c \in E$ such that $\theta \prec \prec c$. Because of $\left\{x_{n}\right\}=$ $\left\{f^{n}\left(x_{0}\right)\right\}$ converges to $x^{*} \in X$ and $f$ is $\perp$-continuous at $x^{*} \in X$, there exists $n_{0} \in \mathbb{N}$ and for all $n \in \mathbb{N}$ such that $n \geq n_{0}$,

$$
d\left(x_{n+1}, x^{*}\right) \prec \prec \frac{c}{2} \text { and } d\left(f x_{n}, f x^{*}\right) \prec \prec \frac{c}{2} .
$$

And so for all $n \in \mathbb{N}$ such that $n \geq n_{0}, d\left(f x^{*}, x^{*}\right) \preceq d\left(f x^{*}, f x_{n}\right)+d\left(f x_{n}, x^{*}\right) \prec \prec c$. On the other hand, for $m \in \mathbb{N}, m \geq 1$ we obtain $0<\frac{1}{m} \leq 1$. Using $c \in$ int $P$ and $\lambda \operatorname{int} P \subseteq \operatorname{int} P(\lambda \in \mathbb{R}, \lambda>0)$ we get $\frac{c}{m} \in \operatorname{int} P$. Thus, for all $n \in \mathbb{N}$ such that $n \geq n_{0}$ and for $m \in \mathbb{N}, m \geq 1$ we hold $d\left(f x^{*}, x^{*}\right) \prec \prec \frac{c}{m}$, then $\frac{c}{m}-d\left(f x^{*}, x^{*}\right) \in P$. Using the cone $P$ is closed set, where taking limit $m \rightarrow \infty$ we get $\lim _{m \rightarrow \infty}\left(\frac{c}{m}-d\left(f x^{*}, x^{*}\right)=\right.$ $-d\left(f x^{*}, x^{*}\right) \in P$. Besides $\theta \preceq d\left(f x^{*}, x^{*}\right)$ that is $d\left(f x^{*}, x^{*}\right) \in P$. So, because of $P$ is cone $d\left(f x^{*}, x^{*}\right)=\theta$ that is $f x^{*}=x^{*}$, so $x^{*} \in X$ is a fixed point of $f$.

Theorem 2.7. Let $(X, \sqsubseteq)$ be a partially ordered set, $(X, \perp, d)$ is an O-complete cone metric space, $f:(X, \perp, d) \rightarrow(X, \perp, d)$ is nondecreasing mapping with respect to $\sqsubseteq$ and $\perp$-preserving. Also suppose that the following conditions hold:

(i) there exists $\alpha, \beta, \gamma \in \mathbb{R}, 0 \leq \alpha, \beta, \gamma$ and $\alpha+2 \beta+2 \gamma<1$ such that

$$
d(f x, f y) \preceq \alpha d(x, y)+\beta[d(x, f x)+d(y, f y)]+\gamma[d(x, f y)+d(y, f x)]
$$

for each orthogonally related $x, y \in X$ with $y \sqsubseteq x$,

(ii) there exists an orthogonal element $x_{0} \in X$ such that $x_{0} \sqsubseteq f x_{0}$,

(iii) if an increasing sequence $\left\{x_{n}\right\}$ converges to $x \in X$, then $x_{n} \sqsubseteq x$ and $x_{n}, x^{*}$ are orthogonally related elements for all $n$.

In this case, the iteration sequence $\left\{f^{n}\left(x_{0}\right)\right\}$ converges to a point $x^{*} \in X$ and $x^{*} \in X$ is a fixed point of $f$.

Proof. As the proof of Theorem 2.6, there exists $x^{*} \in X$ such that $\left\{x_{n}\right\}=\left\{f^{n}\left(x_{0}\right)\right\}$ converges to this point. Now, from the condition (iii) $f^{n}\left(x_{0}\right) \sqsubseteq x^{*}$ and $f^{n}\left(x_{0}\right), x^{*}$ are orthogonally related elements for all $n$. Thus, from the condition $(i)$,

$$
\begin{aligned}
d\left(f x_{n}, f x^{*}\right) \preceq & \alpha d\left(x_{n}, x^{*}\right)+\beta\left[d\left(x_{n}, f x_{n}\right)+d\left(x^{*}, f x^{*}\right)\right]+\gamma\left[d\left(x_{n}, f x^{*}\right)+d\left(x^{*}, f x_{n}\right)\right] \\
\preceq & \alpha d\left(x_{n}, x^{*}\right)+\beta\left[d\left(x_{n}, x^{*}\right)+d\left(x^{*}, x_{n+1}\right)+d\left(x^{*}, x_{n+1}\right)+d\left(x_{n+1}, f x^{*}\right)\right] \\
& +\gamma\left[d\left(x_{n}, x^{*}\right)+d\left(x^{*}, x_{n+1}\right)+d\left(x_{n+1}, f x^{*}\right)+d\left(x^{*}, x_{n+1}\right)\right] \\
= & \alpha d\left(x_{n}, x^{*}\right)+\beta\left[d\left(x_{n}, x^{*}\right)+d\left(x^{*}, x_{n+1}\right)+d\left(x^{*}, x_{n+1}\right)+d\left(f x_{n}, f x^{*}\right)\right] \\
& +\gamma\left[d\left(x_{n}, x^{*}\right)+d\left(x^{*}, x_{n+1}\right)+d\left(f x_{n}, f x^{*}\right)+d\left(x^{*}, x_{n+1}\right)\right]
\end{aligned}
$$

And so, we get

$$
d\left(f x_{n}, f x^{*}\right) \preceq \frac{\alpha+\beta+\gamma}{1-\beta-\gamma} d\left(x_{n}, x^{*}\right)+\frac{2 \beta+2 \gamma}{1-\beta-\gamma} d\left(x_{n+1}, x^{*}\right) .
$$

Let $c \in E$ such that $\theta \prec \prec c$. Because of $\left\{x_{n}\right\}=\left\{f^{n}\left(x_{0}\right)\right\}$ converges to $x^{*} \in X$, there exists $n_{0} \in \mathbb{N}$ and for all $n \in \mathbb{N}$ such that $n \geq n_{0}$,

$$
d\left(x_{n}, x^{*}\right) \prec \prec \frac{c}{2} \frac{(1-\beta-\gamma)}{(\alpha+\beta+\gamma)} \text { and } d\left(x_{n+1}, x^{*}\right) \prec \prec \frac{c}{2} \frac{(1-\beta-\gamma)}{(2 \beta+2 \gamma)} \text {. }
$$

And so for all $n \in \mathbb{N}$ such that $n \geq n_{0}, d\left(f x_{n}, f x^{*}\right) \prec \prec c$. Thus taking limit $n \rightarrow \infty, x_{n+1} \rightarrow f x^{*}$. Using the uniqueness of the limit we obtain $f x^{*}=x^{*}$. 


\section{Conclusion}

In this paper, some basic fixed point theorems are obtained on ordered orthogonal cone metric spaces by considering the continuity and non-continuity of self-mapping. And then generalizations of these theorems are given. Also, an illustrative example is given.

\section{Acknowledgements}

The authors would like to thank the referees for their careful reading and their valuable comments and suggestions to improve their results.

This research article was supported by Amasya University Research Fund Project (FMB-BAP 19-0396).

\section{REFERENCES}

[1] Abbas, M. and Jungck, G., Common fixed point results for noncommuting mappings without continuity in cone metric spaces. Journal of Mathematical Analysis and Applications, 341(1), 416-420,(2008).

[2] Abbas, M. and Rhoades, B. E., Fixed and periodic point results in cone metric spaces. Applied Mathematics Letters, 22(4), 511-515, 2009.

[3] Baghani, H., Gordji, M. E. and Ramezani, M. , Orthogonal sets: The axiom of choice and proof of a fixed point theorem. Journal of Fixed Point Theory and Applications, 18(3), 465-477, 2016.

[4] Bilgili Gungor, N. (2019). Orthogonal cone metric spaces and fixed points of orthogonal contractions. International Journal of Nonlinear Analysis and Applications (In review)

[5] Gordji, M. E., Ramezani, M., De La Sen, M. and Cho, Y. J., On orthogonal sets and Banach fixed point theorem. Fixed Point Theory, 18(2), 569-578, 2017.

[6] Eshaghi Gordji, M. and Habibi, H., Fixed point theory in generalized orthogonal metric space. Journal of Linear and Topological Algebra (JLTA), 6(3), 251-260, 2017.

[7] Huang, L. G. and Zhang, X., Cone metric spaces and fixed point theorems of contractive mappings. Journal of mathematical Analysis and Applications, 332(2), 1468-1476, 2007.

[8] Ilic, D. and Rakocevic, V., Common fixed points for maps on cone metric space. Journal of Mathematical Analysis and Applications, 341(2), 876-882, 2008.

[9] Janković, S., Kadelburg, Z. and Radenović, S., On cone metric spaces: a survey. Nonlinear Analysis: Theory, Methods and Applications, 74(7), 2591-2601, 2011.

[10] Ramezani, M. , Orthogonal metric space and convex contractions. Int. J. Nonlinear Anal. Appl, 6(2), 127-132, 2015.

[11] Ramezani, M. and Baghani, H., Contractive gauge functions in strongly orthogonal metric spaces. Int. J. Nonlinear Anal. Appl, 8(2), 23-28, 2017.

[12] Ran, A. C., and Reurings, M. C., A fixed point theorem in partially ordered sets and some applications to matrix equations. proceedings of the American Mathematical Society, 1435-1443, 2004.

[13] Rezapour, S. and Hamlbarani, R., Some notes on the paper "Cone metric spaces and fixed point theorems of contractive mappings". Journal of Mathematical Analysis and Applications, 345(2), 719-724, 2008.

[14] Turkoglu, D.and Abuloha, M., Cone metric spaces and fixed point theorems in diametrically contractive mappings. Acta mathematica sinica, English series, 26(3), 489-496, 2010.

[15] Vetro, P., Common fixed points in cone metric spaces. Rendiconti del circolo Matematico di Palermo, 56(3), 464-468, 2007.

(NURCAn Bilgili GUngor) Department of Mathematics, Faculty of Science and Arts, Amasya University, 05000, Amasya, Turkey

Email address, NURCAN BILGILI GUNGOR: bilgilinurcan@gmail.com / nurcan.bilgili@amasya.edu.tr

(MEHMET SURMELIOGLU) Department of Mathematics, Institute of Science, Amasya

University, 05100, Amasya, Turkey

Email address, MEHMET SURMELIOGLU: m.surmeli050@gmail.com 